\title{
Ergonomics and sustainability in the design of everyday use products
}

\author{
Francesca Tosi*, \\ University of Florence, Faculty of Architecture
}

\begin{abstract}
The relationship between Ergonomics and Design is a key element in the sustainability project, as well as in many other areas of experimental design. In the Design for Sustainability field, Ergonomics is a strategic factor for design culture innovation, providing designers with the necessary knowledge and skills regarding human characteristics and capabilities, as well as user needs and desires during use and interaction with products in work activities and everyday life. Ergonomics is also a strategic innovative factor in design development and manufacturing processes. In fact, ergonomics provides a methodological approach in user-product interaction evaluation processes through the use of participatory design and survey methods, user trials, direct observation, savings and resource conservation, etc.

On the other hand, design offers solutions able to interpret user needs and expectations, at the same time suggesting new behaviors and lifestyles.

In Design for Sustainability, the ergonomic and user-centered approach contributes greatly to lifestyles and innovative use of products - making it possible to understand and interpret real people needs and expectations in their everyday actions and behavior.

New consumption patterns, new awareness of lifestyles, energy source consumption, purchasing methods and consumption style etc. can be supported by design innovation, responding to expressed and unexpressed user needs. With this in mind, the ergonomic approach represents the starting point for design choices and at the same time, a tool for assessing their appropriateness and effectiveness.
\end{abstract}

Keywords: user needs, lifestyle innovation, product innovation

\section{Introduction}

Ergonomics in Design and Sustainability is one of the most active and innovative components of ergonomic research, and one of the greatest areas of focus in the development of user-involvement based procedures in decision-making and innovation processes.

The development of research lines and areas of experimental design that are transversal to the areas of ergonomics for design and sustainability, springs from a highly innovative approach to the more general theme of the "design for the people" field in which there is a confrontation between research topics and design innovation experiences that frequently tend to find common grounds of exchange and dialogue.
The relationship between Ergonomics and Design is indeed essential in Design for Sustainability, as well as in many other areas of experimental design.

Ergonomics is now an essential component of the design culture, and a key factor for both product and production process innovation, capable of guiding design processes towards the real needs and expectations of individuals and the community.

Ergonomics also provide the necessary methodological contents and an intervention philosophy that allow for building a user-oriented design process and, at the same time, the design offers solutions capable of interpreting user-needs and expectations and suggesting new behaviors and lifestyles. Many of the research and experimentation lines developed in recent years in the field of Ergonomics for

*Corresponding author. E-mail: francesca.tosi@unifi.it 
Design and, in parallel, in the field of environmental sustainability, have addressed from both standpoints the issues of sustainability and safety of production processes and the impact that said processes and more generally, the western development model have on the welfare and health of individuals and the community.

While there are many points of contact among the three areas - ergonomics, design, and sustainability already widely addressed and developed in terms of theoretical and methodological research - the experiments conducted in the field of applied research and experimental design and, in particular, the development of new expressive languages and the creation of innovative design solutions, are instead very recent.

\section{Ergonomics for Design and Sustainability: the development of a research and intervention} area

Many of the contents that have been forming over recent years in the area of Ergonomics in Design, have much in common with the goals and philosophy of sustainable design.

The growing focus on the "people-centered" aspect can be observed in the various design areas and specializations - from product design and architectural design, to town and territorial planning - which are now turning to issues of sustainability of the production and consumption processes, protection of the environment and non-renewable resources generated by the crisis of the western development model.

One of the main features of ergonomics that has characterized its development from the start, is the ability to continually expand its fields of interest and intervention, embracing ever-new disciplines and skills, in an ongoing expansion and enrichment process in which each component - old and new neither excludes nor denies the previous ones, considering them instead as parallel specializations with which to define increasingly new grounds for dialogue and shared survey approaches.

It therefore seems natural for ergonomics to encompass issues of environmental sustainability, to which it can offer, albeit with different intervention methods, its methodological approach aimed at assessing and interpreting the modalities and conditions via which it implements "the interaction between individuals and other elements of a system", and a philosophy of action, oriented to respond with innovative design and intervention solutions to the needs and expectations of the individual and the community.
In the field of Design for Sustainability, as in all fields of design, Ergonomics represents an innovation factor of the design culture that provides the designer with the necessary knowledge about human characteristics and capabilities, and the methodological tools for evaluating different people-needs during use and interaction with the products at work and in everyday life. Ergonomics is also a key factor in the development of design and manufacturing processes. Indeed, it provides structured methods designed to evaluate user-product interaction, and to identify and interpret user needs, expectations and desires.

The ergonomic approach can thus represent both the starting point of the design process - deriving from the knowledge of characteristics and capabilities of Human Factors, and from the knowledge of user needs and expectations, from their interpretation based on structured survey and evaluation methods of the User-Centered Design approach - and at the same time, the tool for assessing their appropriateness and effectiveness.

With regard to the role of design in the field of sustainability, as well as in all its fields of intervention, this is based on the ability to imagine and elaborate innovative design solutions and to synthesize them into a finished product, able to interpret the needs, expectations and desires of people, as well as suggesting new behaviors and lifestyles.

Design is by definition based on the ability to synthesize creative synthesis of innovation and technical feasibility, which may have as its starting point technological and/or production innovations, scientific innovations, factors of social change - or it may spring from the ability to give new interpretations to existing technologies, materials, or production processes, as well as the capacity to interpret existing behaviors, habits, languages and knowledge in an innovative key.

Design is also based on the ability to synthesize different types of disciplinary knowledge - traditionally coming from the scientific, technological and humanistic areas - or rather, the ability to synthesize the knowledge, stimuli and contributions coming from different disciplines and different research areas in the project.

The same training model of the designer is based on the contribution and dialogue between different disciplinary components. A training model that does not produce a "know-all" designer, who knows everything about all the various areas of knowledge in his or her training, but rather, a designer able to tackle the differing research and project topics in a 
complex key through the ability to communicate with all the different disciplines and professional skills, and through the interpretation and systematization of all the contributions, tools and languages. The design approach, at the same time "innovation maker" and interpreter of traditionally far removed knowledge and skills, moves closer to the ergonomics approach and its action philosophy based on the integration of different knowledge and skills, and on a strictly methodological system, capable of providing information and tools for dialogue and comparisons among the various scientific and professional skills.

Similar to ergonomics, the designer also plays a connecting role among different disciplines and skills, with the ability to communicate with other disciplines, skills and also help them communicate amongst themselves. A role of conjunction and synthesis, originating for both from the same system of training pathways based on the multitude of disciplinary sectors involved (and therefore, on knowledge, goals, assessment prospects, methods, experiences, and languages), and on the capacity to tackle each research, intervention and/or project issue from various points of view and on an interdisciplinary basis.

Moreover, Design and Ergonomics work more in terms of problem-solving than abstract theoretical research, characterized by their sector of intervention rather than by their scientific or disciplinary area.

Last, but certainly not least, user centrality - or rather people centrality - is the most relevant aspect in the design and product development process. "User centrality" is the point of contact among the three areas, both in ethical terms, starting from the same definitions of Ergonomics, Design and Sustainability, and in methodological and operational terms.

As E. Manzini and C. Vezzoli in fact state, "(...) the transition to a sustainable society will necessarily consist of the social development of learning, thanks to which, amidst errors and contradictions - as always happens in learning processes - human society will gradually learn to live better by consuming (much) less and by regenerating the quality of its habitat, that is, of the global and local ecosystems and contexts of social life, in which humans have to live." 1

"Designing for the people", i.e. designing for the needs, expectations (and desires) of the people who enter into relationships with objects, buildings, envi-

\footnotetext{
${ }^{1}$ Manzini E., Vezzoli C., Lo sviluppo di prodotti sostenibili, Maggiol, Rimini 1998, 2004
}

ronments, and technological and social systems is the common ground - both theoretical and operational on which Ergonomics, Design, and Sustainability are all based.

A common ground offering a highly innovative approach to the project, which assesses, in a systemic perspective, the various research areas linked to security, usability, welfare, and health, of the three areas of Ergonomics, Design and Sustainability.

3. The construction of an integrated approach to the project: several research experiences in the field of everyday product design

In relation to my research area, the specificity and innovative value of the ergonomic approach to design are in its ability to assess the multiplicity of variables that define the user - product interaction (namely, the features and capabilities of users, the product characteristics and goals, the activities for which it is - or can be - used, and the characteristics of the physical and social context), in turn identifying and interpreting user requirements, expressed or unexpressed, in relation to this interaction.

With particular reference to Design for Sustainability, the ergonomic approach and the UCD approach may contribute greatly to the innovation of lifestyles and also to the way of using everyday products.

The main intervention areas of Ergonomics in Design in the sustainability sector include the field of everyday products and, in general, consumer products, design solutions for social housing and services for the home regarding the sharing of living facilities and of personal services, time management strategies, innovative solutions for energy saving, and conservation of resources, etc.

Design research on some of these issues is currently underway at the Faculty of Architecture of the University of Florence, specifically targeting the development of innovative solutions based on the UserCentered Design approach and aimed at proposing new ways to use everyday products in the domestic and intermodal mobility fields.

The first example involves the project called "Well Living: Ergonomics and Ecology in the kitchen" developed with the EFFETI company in the sector of household kitchens, where the analysis phase of user needs and expectations, allows for identifying areas of technological product innovation - said perfor- 
mance and innovative services can be offered in a mature and consolidated field like that of household kitchens - both in terms of lifestyles and habits regarding the preparation and consumption of food, especially in relation to the proper use and saving of energy sources, waste reduction etc.. The research project was developed in 2009-2010 with joint experimental research and teaching at the Bachelor's Degree of "Design" at the University of Florence, funded by the Region of Tuscany in 2011 .
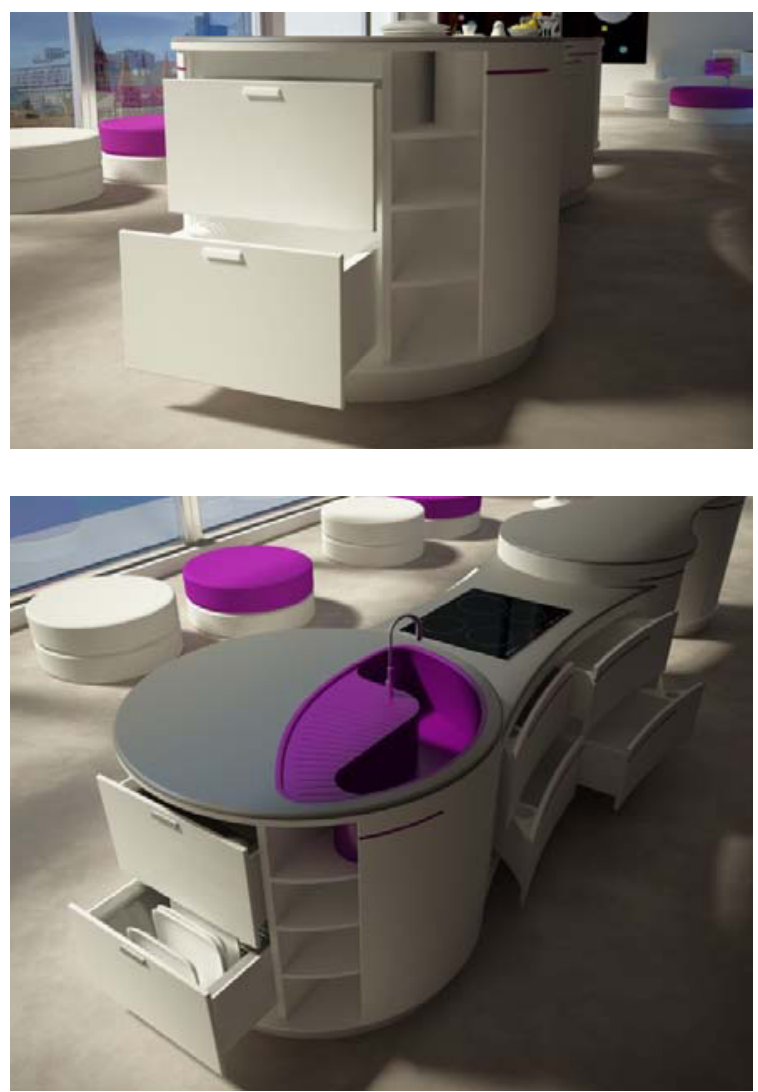

Figure 1-2. Anja - design by Silvia Bicci, Daniele Busciantella Ricci, Giulia Caprili

Workshop "Well Living: Ergonomics and Ecology in the kitchen" developed by: Bachelor's Degree in "Design" Faculty of Architecture, University of Florence with EFFETI company - coordinators: prof. Francesca Tosi, prof. Alessandra Rinaldi

tutors: arch Simone Lucii, dott. Isabella Patti

The second example regards the research project conducted in the field of sustainable mobility and intermodality, "The intermodal bike, Multi-modal integration of cycling mobility through product and process innovations in bicycle design," EU project FP7-SST-2008-RTD -1 - funded in 2010, aimed at creating an easily transportable, folding bicycle in ultra-lightweight material for use on city tracks integrated with the public transport network.

The goal of this program is to develop ergonomic know-how related to the aspects of physiological and psychological "comfort" with specific reference to the compliance of the city/cruising bike with the usability, comfort and biomechanical, anthropometric and physiological compatibility requirements. More specifically, the goals include:

- The definition of a scientifically tested method for measuring and assessing the usability and comfort needs related to bicycles used mainly for urban and intermodal transfers.

- The selection of methods for evaluating the compatibility conditions of anthropometric and postural comfort specifically used for assessing the cycling mobility and the use of bicycles in urban areas.

- The definition of an ergonomic platform (geometric/dimensional matrix) for identifying the best postural trim for a city bike, in order to adapt the different sizes to a single shape and test it on a model.

- $\quad$ The synthesizing of the results in the best possible solution.

- The verification of the validity of the proposed model with tests conducted on models and users.

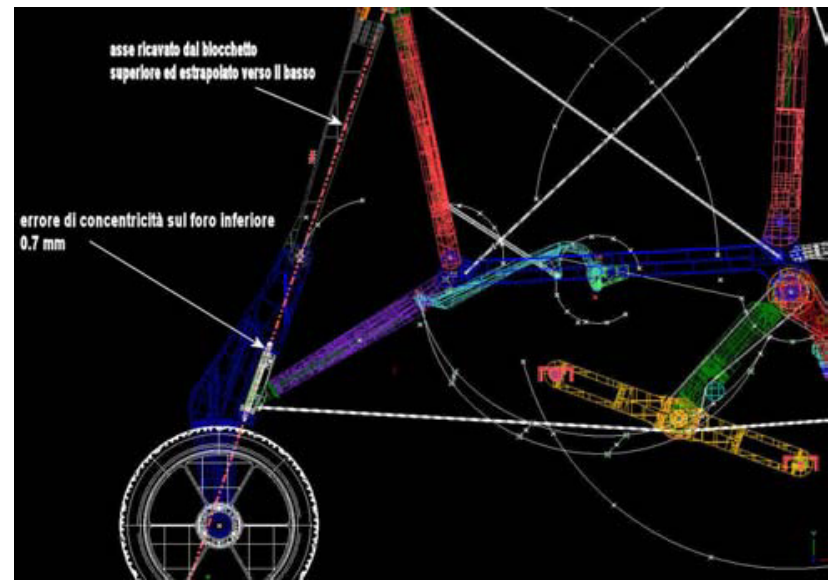




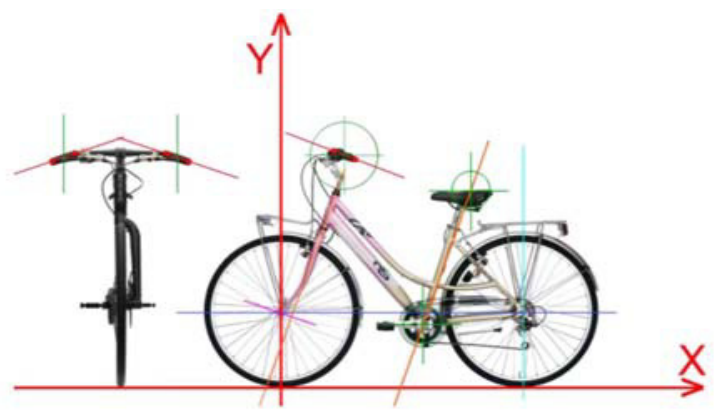

Figure 3-4. THE INTERMODAL BIKE, Multi-modal integration of cycling mobility through product and process innovations in bicycle design - FP7-SST-2008-RTD-1 2010-2013

Partners:

1. Tecnologie Urbane Srl TECNO Italy

2. Ticona GmbH TICONA Germany

3. University of Florence UNIFI Italy

4. TRILIX Car Design TRILIX Italy

5. MAXON Motors MAXON Switzerland

6. ATAF Florence ATAF Italy

7. LPP Ljubljana LPP Slovenia

Program scientific coordinator: arch. Alessandro Belli scientific coordinator for University of Folrence unit: prof. Francesca Tosi, with: prof. Giulio Arcangeli, prof. Grazia Tucci, arch. Alessandra Rinaldi, dott. Marco Petranelli

\section{References}

[1] L. Bandini Buti, Ergonomia olistica, il progetto per la variabilità umana, Franco Angeli, Milano 2008

[2] P.Bertola, E.Manzini, Design Multiverso, Polidesign, Milano 2006

[3] E.Manzini, F. Jegou, Quotidiano sostenibile. Scenari di vita urbana, Ambiente, Milano 2003

[4] E. Manzini, C. Vezzoli, Lo sviluppo di prodotti sostenibili, Maggioli, Rimini 1998, 2004

[5] G. Mantovani, Ergonomia, lavoro, sicurezza e nuove tecnologie, Il Mulino, Bologna 2000

[6] D. Norman, Emotional Design, Basic Books, New York, 2004

[7] D. Norman, Living with complexity, The MIT Press, Cambridge, Massachusetts, 2010

[8] F. Tosi, Ergonomia, progetto, prodotto, Franco Angeli, Milano 2005

[9] F. Tosi, Ergonomia e progetto, Franco Angeli, Milano 2006

[10]I. McClelland, "Product assessment and user trials", in: Wilson J.R. e Corlett E.N., Evaluation of Human Work, Taylor \& Francis, Londra - Philadelphia 1995

[11]R. Verganti, Design driven innovation, Etas, Milano 2009

[12]J. Zink Klaus, Corporate sustainability as a challenge for comprehensive management, Physica-Verlag, 2008 